\title{
Quelques résultats d'enquêtes récentes sur la globidiose du dromadaire au Tchad* Note préliminaire
}

\author{
par J. GRUVEL ef M. GRABER
}

\begin{abstract}
RÉSUMÉ
La Coccidiose cameline ou Globidiose due à Globidium cameli HENRY et MASSON 1932 est une affection parasitaire dont la connaissance est relativement récente.

Des examens coprologiques réalisés au cours d'enquêtes helminthologiques pratıquées sur quelques effectifs camelins ont permis de mettre en évidence ce parasitisme au Tchad. Celul-ci est assez faible puisque sur 204 examens, 14 seulement révélaient la présence du Globidıum.

L'importance de ce parasitisme n'apparaît pas primordıale mais celui-ci jove néanmoins son rôle en participant au poly-parasitisme si fréquent chez le dromadaire.

Le texte est accompagné d'un tableau, de 2 cartes ef d'une bibliographie comprenant 6 références.
\end{abstract}

La pathologie du dromadaire est dominée au Tchad par les maladies parasitaires : trypanosomiases, gales, helminthiases. A ces dernières s'associe bien souvent la Coccidıose à Globidium comeli HENRY ef MASSON 1932.

La Coccidiose camelıne due à Globıdium cameli est une affection parasitaire connue depuis relativement peu de temps. En 1920, DOBERTY décrivait au Kenya une maladie des dromadaires dont la cause peut être imputée à ce parasite. Mais c'est seulement en 1932 que HENRY et MASSON devaient découvrir et décrire cette coccidie spécifique du dromadaire chez un animal du Jardin des Plantes à Paris. L'autopsie révéla des lésions uniquement au niveau de l'intestin grêle sous la forme d'un piqueté blanchâtre dans lequel le parasite fut découvert.

Quelques enquêtes helminthologiques effec-

(*) Communıcation présentée à la $2^{\mathrm{e}}$ Conférence Internationale de prolozologie (Londres, 29 juiliet-5 août 1965). tuées au Tchad ces dernières années permirent de mettre en évidence ce type de Coccidie et montrent l'intérêt que peut présenter ce parasitisme.

L'élevoge du dromadaire, fort de 500.000 têtes environ, se situe dans tout le territoire du Tchad situé au nord du $13^{\mathrm{e}}$ parallèle où les précipifations annuelles sont constamment inférieures à $500 \mathrm{~mm}$. En raison des déplacements d'animaux de transport, quelques dromadaires peuvent cependant être encore rencontrés plus au sud, mais sans dépasser une limıte déterminée par une ligne joignant Massakory à Goz-Beida (voir carte).

Des examens coprologıques on été effectués systématiquement sur 192 chameaux provenant du Kamen ou du Ouaddaï, appartenant à des troupeaux de grande nomadisation ou d'élevages plus localisés ; 8 d'entre eux ont été trouvés fortement parasités par Globidum cameli. Très récemment (juillet 1965) 12 dromadaires 


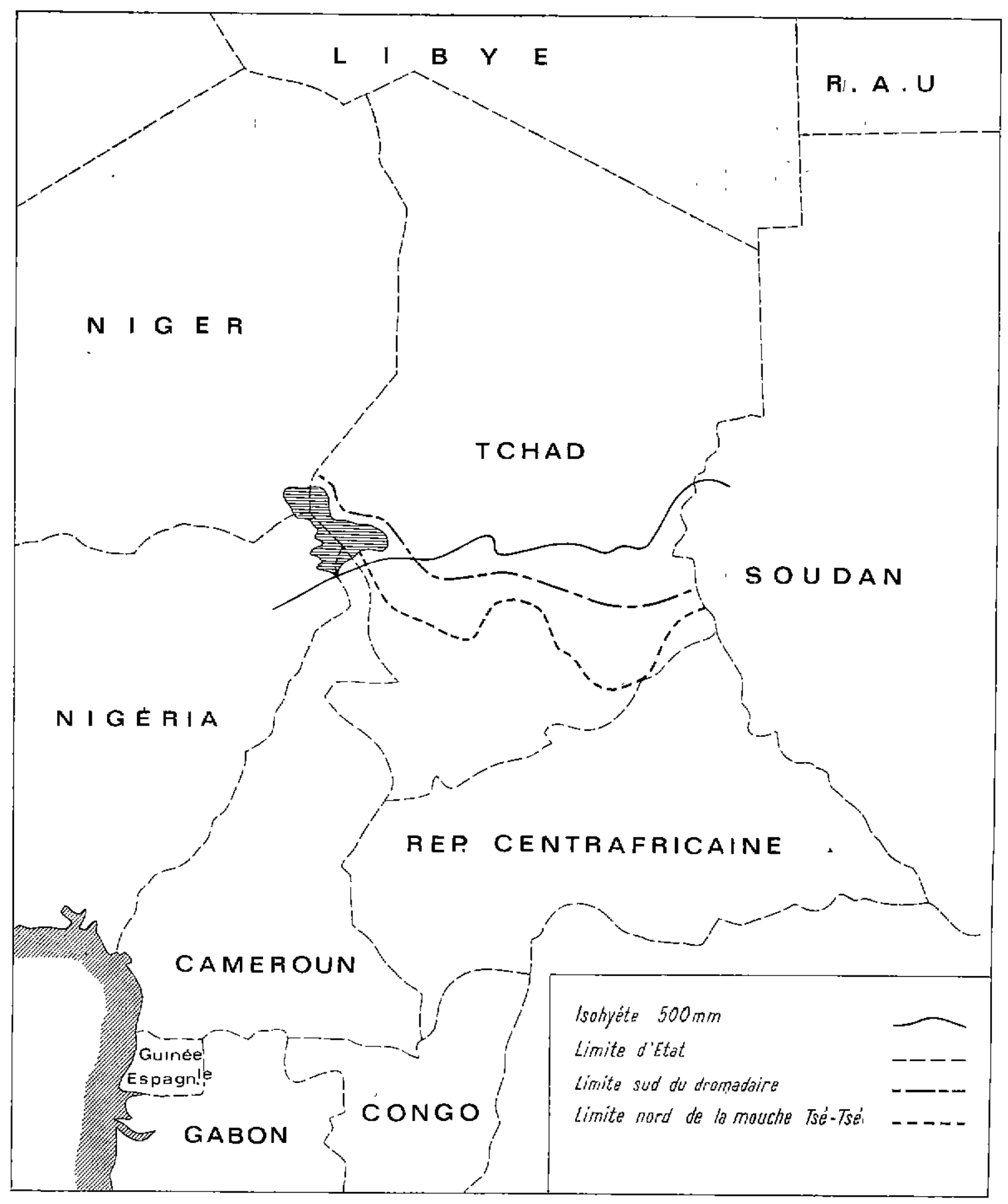




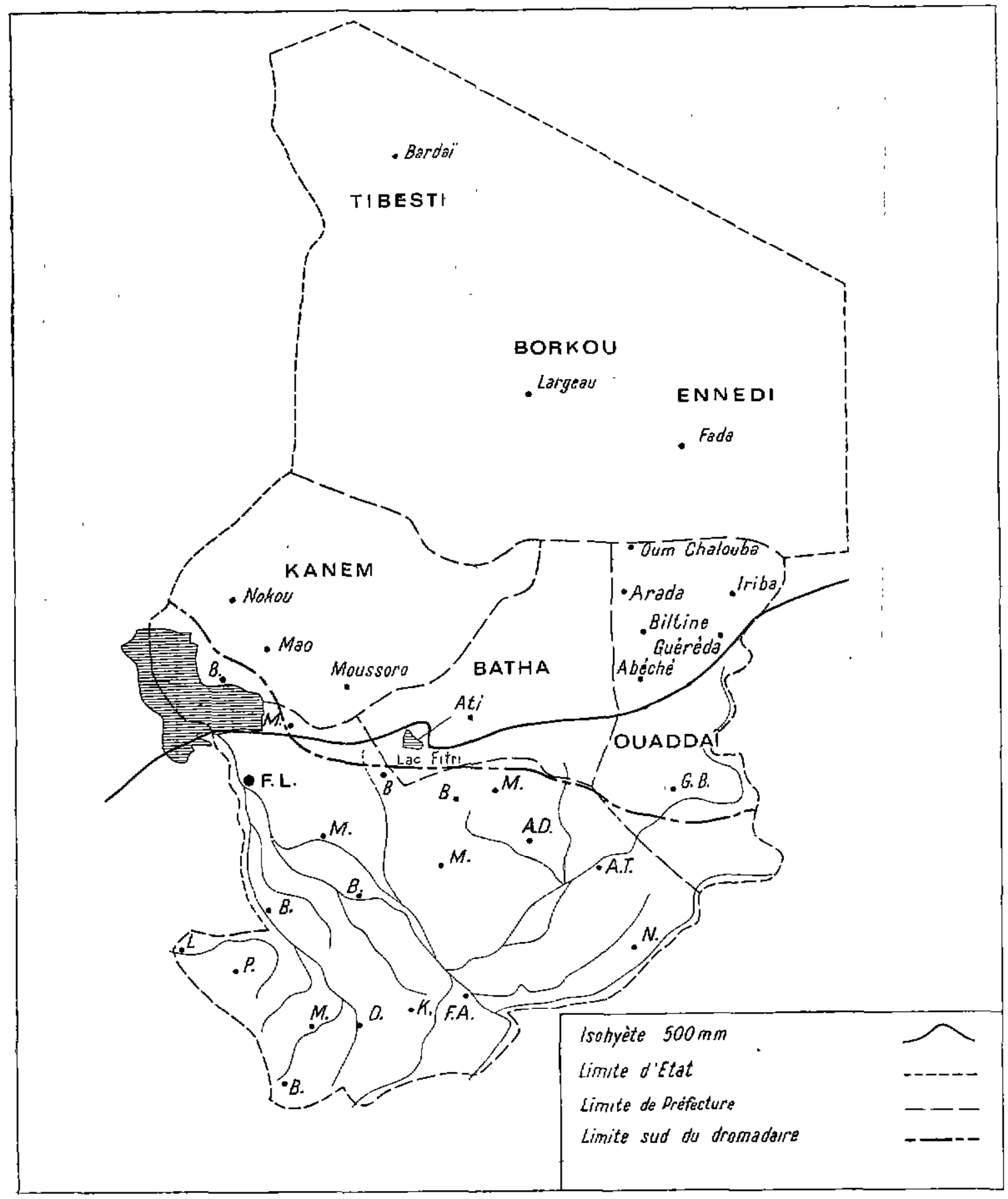




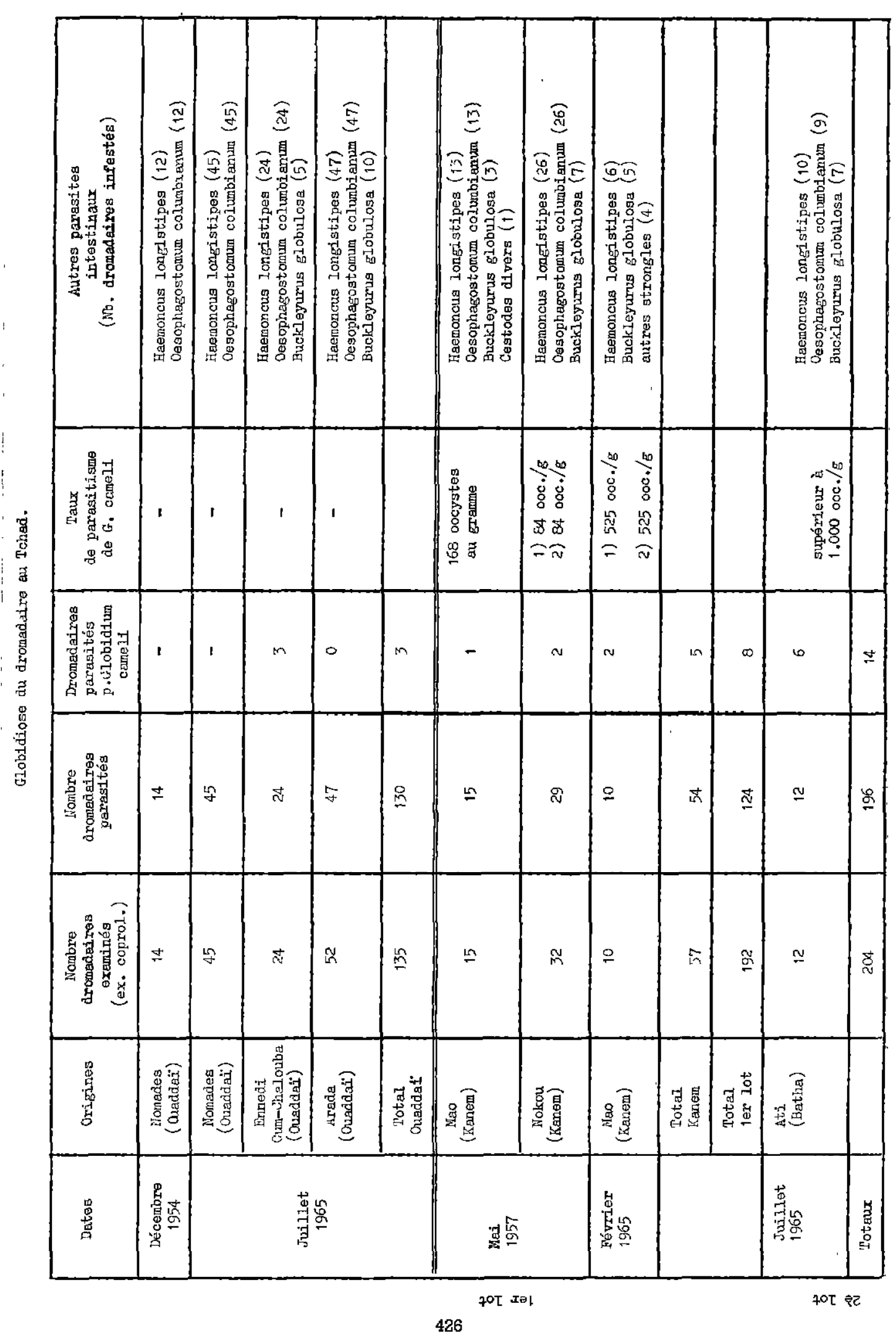


venant d'Ati (Préfecture du Batha), ont été examinés au Laboratoire de Farcha et 6 trouvés porteurs de Globidium.

Ainsi, sur 204 dromadaires originaires des 3 provinces constituant le sud de la zone d'élevage, 14 étaient infestés. Dans le premier lot, les taux d'infestation varıaient de 84 à 525 oocystes au gramme; dans le second, quelques examens donnèrent plus de 1.000 oocystes au gramme et même 1.260 pour l'animal le plus parasité, très maigre ef dont l'autopsie révéla de très abondantes léssons sur toute la longueur de l'intestin grêle.

Les enquêtes sont encore trop fragmentaires pour que l'on puisse attribuer à ce parasitisme une importance fondamentale ; mais il apparaît indéniable qu'à lui seul il pourrait provoquer des troubles altérant la santé de l'animal et le rendant vulnérable à d'autres maladies. Les taux relevés au cours des examens semblent supportés par les animaux. Cependant, le dromadaire le plus parasité, avec des taux variant de 500 à 1.260 oocystes au gramme selon les examens, était extrêmement maigre. Une action nuisible du Globidium pourrait donc être possible à partır d'un certain seuil.

Ce parasitisme rarement signalé est le plus souvent associé à de nombreux helminthes intestinaux dont les plus fréquemment rencontrés sont Hoemoncus longistipes RAILLET ef HENRY 1909. Oesophagostomum columbianum CURTICE 1890 ef Stilesia globipunctoto RIVOLTA 1874. II semble d'ailleurs qu'existe une certaine compétition entre la Coccidiose et l'Helminthiase. Nous avons en effet remarqué que les dromadaires les plus fortement parasités par $G$. cameli montraient des taux d'infestation par les Helminthes relativement bas.

Le développement de G. comeli contribue de toute manière au polyparasitisme préjudiciable à l'équilibre sanitaire d'un troupeau ef pouvant causer dans certains cas une mortalité importante variant de 5 à 10 p. 100 selon les régions ef les années.

L'intérêt de ces observations réside dans le fait que ce parasitisme n'a pratiquement jamais été signalé en Afrique Centrale et que son rôle pathogène est mal connu.

Une étude plus complète de la fréquence, de la répartition de ce parasite doit succéder à ces enquêtes préliminaires et préciser la juste part qu'il convient de lui attribuer dans la pathologie du dromadaire.

\section{CONCLUSION}

Les enquêtes helminthologiques récentes pratıquées sur des effectifs camelins du nord-Tchad ont mis en évidence la présence de Globidium cameli HENRY et MASSON 1932, parasite spécifique du dromadaire.

Institut d'Elevage ef de Médecine vétérnoire
des Pays tropicaux.
Laboratoire de Farcha-Fort-Lamy (Tchad)

\section{SUMMARY}

Résults of a recent survey on camel globidiosis in Chad. Preliminar note.

Camel coccidiosıs or globidiosis caused by Globidum cameli HENRY and MASSON 1932 is a parasitic disease known ralher recently.

Examination of feces carried out during nelminthologic surveys in camels allowed to show this parasitism in Chad. Since only 14 examinations out of 204 revecled the presence of Globidium, this parasitism does not seem very important, but yet it plays its part participating in polyparasitism so frequent in the dromedary.

One table, Iwo maps and a bibliography with six references accompany this paper. 


\section{RESUMEN}

Algunos resulfados de encuestas recientes sobre la globidiosis del dromedario en el Chad. Nota preliminar.

La coccidiosis de los camellos o globidiosis por Globidium cameli HENRY y MASSON 1932 es una enfermedad parasitaria conocida desde hace relativamente poco tiempo.

Examenes coprológicos realizados durante encuestas helmintológicas efectuadas en camellos permitieron demostrar este parasitismo en el Chad. Este es demasiado poco impartante ya que entre 204 examenes solo 14 revelaban la presencia del Globidium.

La importancia de este parasitismo no parece primordial pero sin embargo este desempeña su papel, teniendo parte al poliparasitismo tan frecuente en el dromedario.

Un cuadro, 2 mapas y una bıbliografia con 6 referencias acompañan este documento.

\section{BIBLIOGRAPHIE}

1. DOBERTY. - British East Africa, Departement of Agriculture. Annal Report, 1909-1910, p. 42.

2. ENICK (K.). - Zur Kenntnis des Globidium cameli und der Eimeria cameli. Arch. Protisenk, $1933,83: 371-80$.

3. HENRY (A.) et MASSON (G.). - Sur une forme coccidienne de l'intestin du chameau. Comp. Rend. Séances Société de Biologie, $\mathrm{ClX}$ $1932 ;$ I : 17-8.
4. HENRY (A.) et MASSON (G.). - Considérations sur le genre Globidium, Globidium cameli, n. sp., parasite du dromadaire. Ann. Paras. Hum. et comparée, 1932, X. 5 : 385-401.

5. HENRY (A.) et MASSON (G.) - La coccidlose du dromadaire. Rec. Méd. Vét. Exol., $1932: 185$.

6. YAKIMOV (V. L.). - Z Zur Frage der Coccidien der Kamela. Arch. Wiss. Prakt. Tierheilk, 1934, $68: 134-7$. 